\title{
Continuous transition between decagonal quasicrystal and approximant by formation and ordering of out-of-phase domains
}

\author{
M. Döblinger, R. Wittmann,* and D. Gerthsen \\ Laboratorium für Elektronenmikroskopie, Universität Karlsruhe, Kaiserstrasse 12, D-76128 Karlsruhe, Germany \\ B. Grushko \\ Institut für Festkörperforschung, Forschungszentrum Jülich, D-52425 Jülich, Germany
}

(Received 29 November 2001; published 20 May 2002)

\begin{abstract}
The transformation between a quasicrystal and an orthorhombic approximant is studied at the nominal composition $\mathrm{Al}_{72.7} \mathrm{Ni}_{8.3} \mathrm{Co}_{19}$ by electron diffraction and high-resolution transmission electron microscopy. A series of transition states indicating a continuous transformation is monitored. First, the material transforms to a single-oriented one-dimensional quasicrystal. In the course of this process out-of-phase domains are formed. The approximant results from ordering of these domains to a periodic structure.

DOI: 10.1103/PhysRevB.65.224201

PACS number(s): 61.44.Br, 61.50.Ks, 68.37.Lp
\end{abstract}

\section{INTRODUCTION}

Quasicrystals are structures with long-range aperiodic order that may exhibit noncrystallographic rotational symmetries. They are observed in many alloy systems as stable phases, most of them with icosahedral or decagonal symmetry. ${ }^{1}$ Most of these alloy systems show periodic phases whose structure is closely related to quasicrystals, so-called approximants. The transition between quasicrystal and approximant involves a rearrangement of atom positions from aperiodic to periodic while the building units are almost identical. ${ }^{2}$ Quasicrystals and approximants can be described in a higher-dimensional approach as embedded into a periodic hypercrystal structure. ${ }^{3,4}$ The quasiperiodic structure in physical space results from a hyperplane that cuts the hypercrystal with an irrational slope. Changing the slope of the hyperplane or the shape of hyperatoms implicates different arrangements in physical space. The lattice of crystalline approximants can be derived from the quasicrystal by so-called linear phason strain which corresponds to a shear of the hyperplane to a rational slope. The corresponding diffraction patterns show reflections shifted from quasiperiodic arrangements to periodic arrangements.

States corresponding to phason strain values between decagonal quasicrystal and one-dimensional quasicrystal (1D QC) were observed by electron diffraction ${ }^{5}$ in material directly quenched from the melt. A transition by continuously increasing linear phason strain with domain sizes exceeding the nanometer range was suggested. In contrast to these untwinned transition states are the frequently observed structures built of twinned phason strained domains in the range of nanometers, called nanodomain structures (NDS's). ${ }^{6,7}$ The origin of twinning is assumed to be related to the misfit between a periodic and quasiperiodic structures increasing with the size of the transformed region. These NDS's are not necessarily periodic in three dimensions, multiply twinned $1 \mathrm{D} \mathrm{QC'} \mathrm{s}^{8}$ or states whose order according to a $1 \mathrm{D}$ QC is only partially developed were also observed. ${ }^{9}$ There is still a lack of experimental data about the transition of NDS's to larger domains of periodic approximants.

At present most of the known periodic approximants to quasicrystals related to fivefold or tenfold symmetry are so- called Fibonacci approximants. ${ }^{10}$ The Fibonacci series is given by the recursion $F_{k+1}=F_{k}+F_{k-1}$, starting with $F_{0}$ $=0$ and $F_{1}=1$. In the limit, the ratio of two consecutive numbers of this sequence is $\tau=(1+\sqrt{5}) / 2$, the golden mean. The Fibonacci approximants can be regarded as constituents of a sequence whose limit is a quasicrystal. The lattice constants in the pseudodecagonal plane can be derived within this view from the quasilattice constants. ${ }^{10,11}$ In the diffraction pattern, the most prominent reflections of Fibonacci approximants are given by indices belonging to the Fibonacci numbers or their integer multiples. An approximant that does not show these features was observed in the system Al-Fe-Cr and named "non-Fibonacci approximant." 12,13

The formation of antiphase domains can be observed in the process of phase transformations. ${ }^{14}$ Antiphase domains can be regarded as a special case of out-of-phase domains. In this case, the phase shift of diffracted waves is not necessarily $\pi$; also, other fractions of $2 \pi$ than 0.5 are allowed as phase shifts. For quasicrystals the concept of out-of-phase domains can be extended to a description in higherdimensional space. ${ }^{15}$ An illustrative and widely discussed example for periodic arrangements resulting from the formation of antiphase domains is the case of CuAu-II. ${ }^{16-18}$ With respect to the aristophase $\mathrm{CuAu}-\mathrm{I}$, the reflections are split into a pair of new ones.

In the system Al-Ni-Co a number of quasicrystalline variants of the decagonal phase are observed. ${ }^{19-21}$ For many compositions annealing as-cast material about 100-200 K below the solidus results in the formation of periodic approximants. ${ }^{10,22,23}$ NDS's were observed in the system AlNi-Co by high-resolution x-ray diffraction ${ }^{8,24,25}$ and highresolution transmission electron microscopy (HRTEM). ${ }^{9}$ At compositions close to the binary Al-Co line the phase diagram seems to be very complicated. ${ }^{26}$ Pentagonal quasicrystals are reported ${ }^{27,28}$ to exist between $\mathrm{Al}_{73} \mathrm{Ni}_{7} \mathrm{Co}_{20}$ and $\mathrm{Al}_{72.5} \mathrm{Ni}_{10} \mathrm{Co}_{17.5}$. The high-temperature modification exhibits so-called S1-superstructure reflections while the lowtemperature ordering state (below about $1170 \mathrm{~K}$ ) does not. $\mathrm{An}$ in situ study by $\mathrm{x}$-ray diffraction $^{29}$ revealed at $\mathrm{Al}_{73.5} \mathrm{Ni}_{4.8} \mathrm{Co}_{21.7}$ a primitive quasicrystal as high-temperature 
phase while the material transformed to an approximant at lower temperatures. Another approximant was described by Hiraga et al. ${ }^{35}$ around $\mathrm{Al}_{72} \mathrm{Ni}_{7} \mathrm{Co}_{21}$.

In the current work we report a continuous transition of an Al-Ni-Co decagonal phase to a "macroscopic" periodic structure resulting from a mechanism governed by increasing phason strain as well as formation and ordering of out-ofphase domains.

\section{PHASON STRAIN}

Phason strain analysis is a useful tool for the identification of transition states of quasicrystals as a measure for the deviation of a tiling from ideal quasicrystalline order. Tilings from HRTEM images and electron diffraction patterns taken with small selected-area apertures can give local information on phason strain of single domains. Periodic approximants and 1D QC's correspond to special values of linear phason strain with respect to a two-dimensional quasicrystal. For reviews of higher-dimensional crystallography, see Yamamoto $^{30}$ or Steurer and Haibach. ${ }^{11}$

Each vertex of a decagonal tiling with edge length $a$ can be indexed unambigiously as linear combinations of four vectors $\mathbf{e}_{i}=a(\cos (2 \pi i / 5), \sin (2 \pi i / 5)), i=1, \ldots, 4$, resulting in the coordinates $\mathbf{r}_{\|}=\Sigma_{i} n_{i} \mathbf{e}_{i}$, where $n_{i}$ are integers. For practical means it is sometimes more convenient to use five linear dependent vectors $\mathbf{e}_{i}=a(\cos (2 \pi i / 5), \sin (2 \pi i / 5)), i$ $=0, \ldots, 4$. It can be reduced to the description by four vectors using the relation $\left(m_{0}+n, m_{1}+n, m_{2}+n, m_{3}+n, n\right)$ $=\left(m_{0}, m_{1}, m_{2}, m_{3}, 0\right)$, where $m_{i}$ are integers. The vectors $\mathbf{e}_{i}$ can be regarded as projections of a basis of a fourdimensional lattice. This four-dimensional space can be divided into physical space (tiling) and perpendicular space with $\mathbf{r}_{\perp}=\sum_{i} n_{i} \mathbf{e}_{2 i}$. The projection of all vertices of an ideal quasiperiodic tiling into perpendicular space (perpendicular projection) must lie within so called acceptance domains $A_{p}$, i.e., $\left\{\mathbf{r}_{\perp}\right\} \subset A_{p}$, which can be different depending on $p$ $=\left(\sum_{i} n_{i}\right)_{\bmod 5}$. The type of the quasiperiodic tiling determines size and shape of the acceptance domains. If a tiling deviates from ideal quasiperiodicity, there are also projected vertices outside of the acceptance domain. Statistical deviations from quasiperiodicity leading to an isotropic distribution of projected vertices are random phason fluctuations (around the average phason strain $\mathbf{E}=0$ ). In the case of linear phason strain, the distribution of vertices in perpendicular space is anisotropic. $\mathbf{E}$ is then given by a $2 \times 2$ matrix in the case of a two-dimensional quasicrystal. For an infinite extension of a tiling with linear phason strain, the projection of the vertices extends infinitely in perpendicular space. An interesting method to analyze linear phason strain in experimental tilings using an inclined projection of vertices is described by Ritsch et al. ${ }^{31}$ By this method the vertices can be reprojected into the acceptance domain by applying a reverse transformation corresponding to the present or expected linear phason strain: $\mathbf{r}_{\text {incl }}=\mathbf{r}_{\perp}-\mathbf{E} \mathbf{r}_{\|}$. By this means one can check if a tiling shows a certain value of linear phason strain. As a scale, we use the decagonal acceptance domain of a so-called Penrose pentagon tiling in evaluations of experimental tilings in Sec. IV B. With respect to a $2 \mathrm{~nm}$ tiling, high-quality variants of a one-dimensional quasicrystal and superstructure $\mathrm{S} 1$ can be projected into this acceptance domain. ${ }^{9,31}$

In the diffraction pattern linear phason strain causes peak shifts. ${ }^{32}$ Thus $\mathbf{E}$ can be determined according to $\mathbf{k}_{\|}=\mathbf{k}_{i d}$ $+\mathbf{E}^{\dagger} \mathbf{k}_{\text {perp }}$, where $\mathbf{k}_{i d}$ corresponds to the peak position of an ideal quasicrystal which is shifted by the transpose of $\mathbf{E}$ to the measured peak position $\mathbf{k}_{\|}$.

\section{EXPERIMENT}

Ingots of nominal composition of $\mathrm{Al}_{72.7} \mathrm{Ni}_{8.3} \mathrm{Co}_{19}$ were prepared by inductive melting in a water-cooled copper crucible under an Ar atmosphere. Different samples were homogenized for $40 \mathrm{~h}$ at 1300,1310, and $1325 \mathrm{~K}$ in an alumina crucible under Ar atmosphere and subsequently quenched. The different homogenization temperatures are used for the quenched samples to make sure that the material is annealed sufficiently close to the solidus temperature. For comparison, material homogenized at $1300 \mathrm{~K}$ for $40 \mathrm{~h}$ was furnace cooled. Parts of the quenched samples were annealed at 1120 $\mathrm{K}$ for $120 \mathrm{~h}$ (after homogenization at $1310 \mathrm{~K}$ ) and $1370 \mathrm{~h}$ (after homogenization at $1300 \mathrm{~K}$ ) in evacuated silica ampoules. After each heat treatment the samples were investigated by scanning electron microscopy (SEM) and powder $\mathrm{x}$-ray diffractometry. The SEM examinations did not reveal any contrast which could be associated with compositional inhomogeneity. The powder x-ray diffraction patterns were typical of the decagonal phase; the resolution was not sufficient to observe the structural variations resulting from different annealing procedures (see below). No additional reflections associated with the formation of neighboring phases were observed. The average composition was determined by wavelength dispersive spectrometry (Cameca camebax SX 50) to $\mathrm{Al}_{72.9} \mathrm{Ni}_{7.9} \mathrm{Co}_{19.2}$ with maximum deviations of 0.5 at. \%. HRTEM and electron diffraction was carried out with a Philips CM 200 ST/FEG $\left(C_{s}=1.2 \mathrm{~mm}\right)$ microscope operating at $200 \mathrm{kV}$ on powdered specimens supported on copper grids covered by holey carbon film. HRTEM images are recorded close to the Scherzer defocus $(-64 \mathrm{~nm})$.

If not indicated otherwise, selected-area apertures with a diameter of $760 \mathrm{~nm}$ are used for recording diffraction patterns. For special purposes, small selected-area apertures with a diameter of only $170 \mathrm{~nm}$ are used. ${ }^{33}$

\section{EXPERIMENTAL RESULTS}

\section{A. Electron diffraction}

A phase analysis of quenched material homogenized at different temperatures shows no qualitative differences. The major part of the material exhibits electron diffraction patterns very similar to those associated in the literature with the primitive fivefold variant (" $5 f$ ") (Refs. 21 and 27) of the decagonal phase [Figs. 1(c) and 1(d)]. Diffraction patterns of these states recorded at thick sample regions suggest a fivefold symmetry like in Fig. 3(d). ${ }^{34}$ The S1 superstructure shown in Fig. 1(a) was observed only as a minority phase in material quenched after homogenization. The material annealed at $1300 \mathrm{~K}$ and subsequently furnace cooled contained a disordered approximant. The same approximant, to a con- 


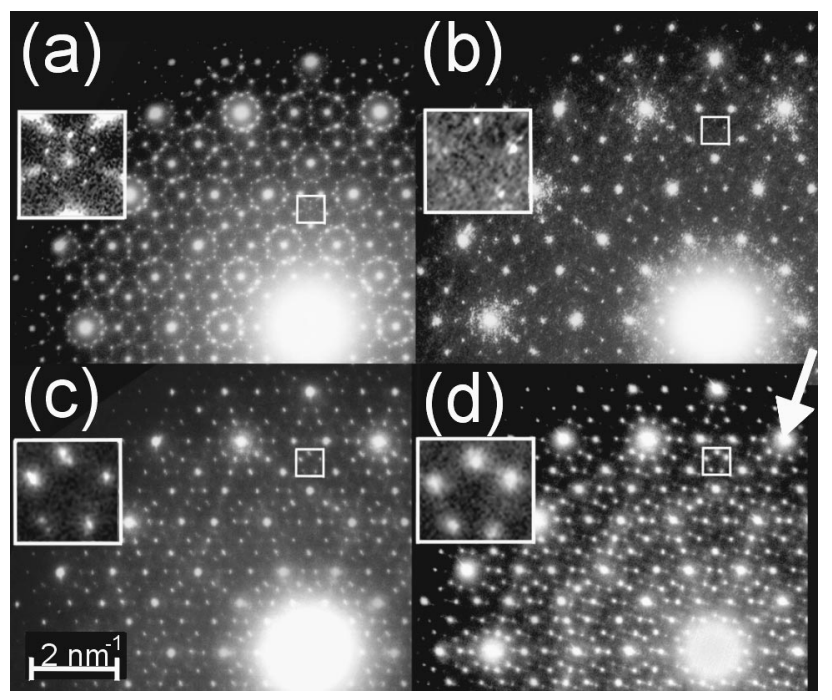

FIG. 1. Diffraction patterns of material quenched after homogenization at $1300 \mathrm{~K}$ (c) and $1310 \mathrm{~K}$ (a), (b), and (d). (a) S1 superstructure. The magnified detail shows that highly indexed reflections are slightly shifted from a symmetric pentagonal arrangement. (b) Diffraction patterns of small sample area next to the position of diffraction pattern (a). The reflections are diffuse and shifted from positions of a decagonal quasicrystal. (c), (d) Diffraction patterns of larger sample areas. The reflections are clearly shifted. In the case of (d), the reflection positions almost correspond to a 1D QC. The arrow shows the periodic direction. Additionally elongated reflections and streaks are recognizable.

siderable part well ordered, was found in the samples preannealed at $1300 \mathrm{~K}$, subsequently quenched, and additionally annealed at $1120 \mathrm{~K}$ for $120 \mathrm{~h}$ and $1379 \mathrm{~h}$ [Fig. 3(b)]. From these observations we can conclude that under the studied conditions a single decagonal phase solidified from the liquid transformed to a single periodic structure.

In some diffraction patterns of the S1 superstructure weak reflections are slightly shifted which indicates small amounts of phason strain as is the case for Fig. 1(a). In comparison to the $\mathrm{S} 1$ superstructure at compositions containing more $\mathrm{Ni}$, the superstructure reflections are less sharp. This effect is also visible on diffraction patterns of the S1 superstructure shown by other authors ${ }^{28,35}$ at similar compositions. It was observed that the S1 superstructure appears in the same grain next to states with diffraction patterns like in Fig. 1(b). While reflections shifted from pentagonal or decagonal symmetry are rather an exception in the case of the S1 superstructure this is always observed for states without superstructure reflections. These phason strained samples therefore belong to transition states of a quasicrystal. Examples are shown by the enlarged details of Fig. 1. In Fig. 1(d) the phason strain corresponds almost to a $1 \mathrm{D}$ QC. The periodic orientation is marked by an arrow. All other diffraction patterns are shown in the same orientation. Some of the reflections in this diffraction pattern are strikingly elongated. In addition, weak streaks connecting single reflections are recognizable.

The diffraction patterns in Fig. 2 are presented in a sequence where the features of a quasiperiodic structure are gradually replaced by those of the periodic structure.

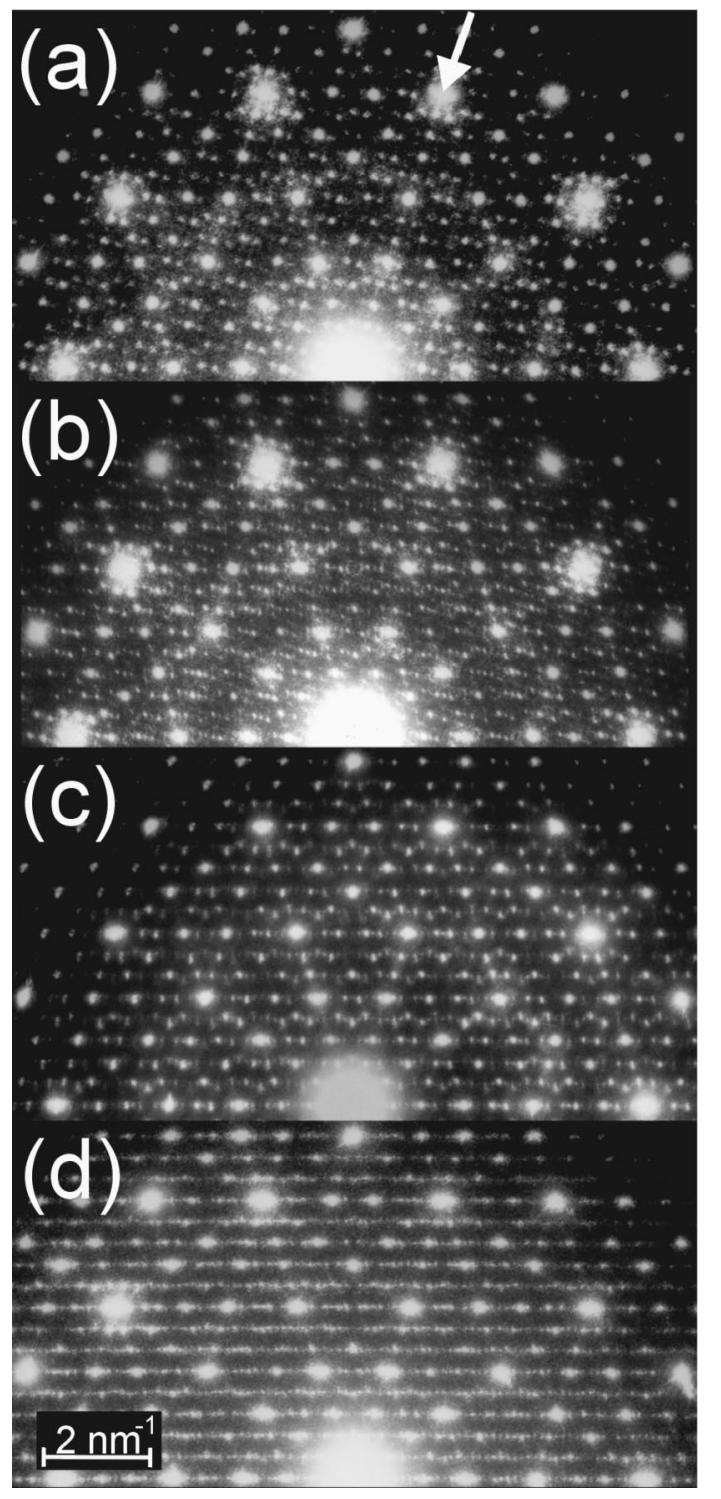

FIG. 2. Diffraction patterns of material quenched after homogenization from $1310 \mathrm{~K}$ (a), (b), (d), and $1325 \mathrm{~K}$ (c). (a) Onedimensional quasicrystal. (b) Ordering state exhibiting apart from the reflections like in (a) additional reflections. The arrangement resembles to a sawtooth pattern. (c) The reflections form a zigzag pattern. It looks as if the reflections are approaching horizontal lines. (d) The reflections are arranged almost exactly on horizontal lines corresponding to a distance of $3.2 \mathrm{~nm}$. The main reflections are strongly elongated in the horizontal direction.

When small selected-area apertures are used for sample positions as represented by diffraction pattern of Fig. 1(d), two different kinds of diffraction patterns are observed. One kind corresponds to a 1D QC [Fig. 2(a)]. The other sort of diffraction patterns also reveal reflection positions of a 1D QC. However, some of the reflections are clearly elongated like in Fig. 2(b). There are also additional reflections arranged on diagonal lines. Altogether, a sawtooth pattern of reflections arises. As the selected area aperture was increased in size the weaker sharp reflections merged into a streak [Fig. 1(d)]. This shows that the underlying structural features are correlated over larger distances only with respect to their 


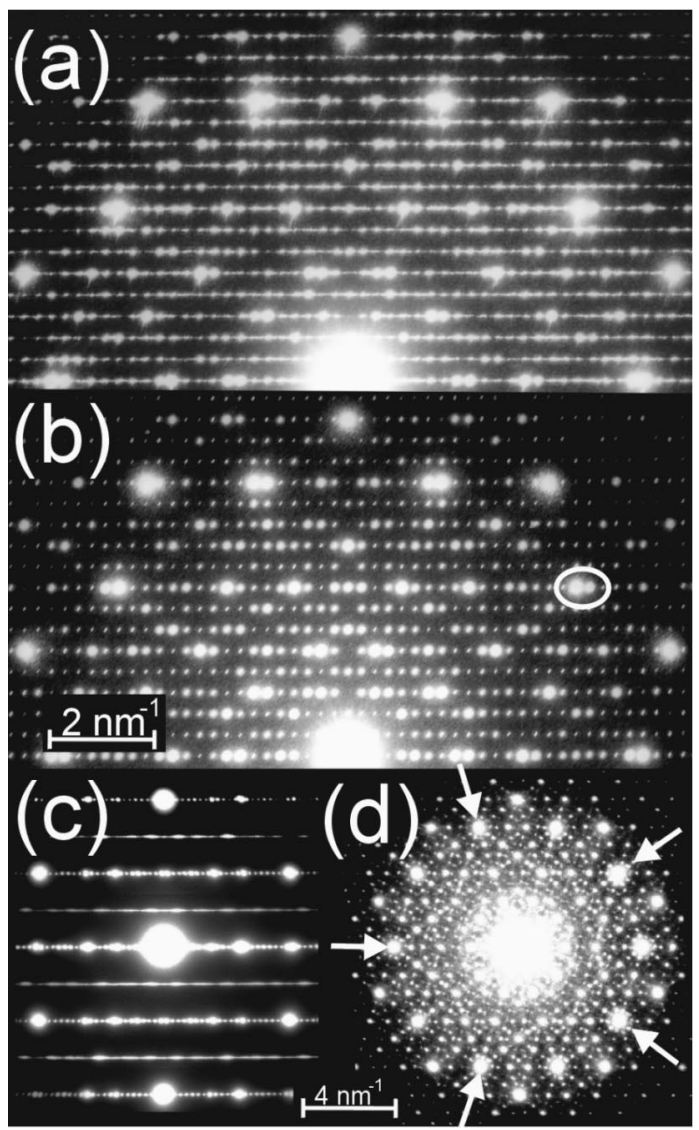

FIG. 3. (a) Diffraction pattern of an approximant exhibiting streaks in the horizontal direction. It comes from material that was furnace cooled after homogenization at $1300 \mathrm{~K}$. (b) High-quality approximant obtained from annealing for $1370 \mathrm{~h}$ at $1120 \mathrm{~K}$ after homogenization at $1300 \mathrm{~K}$. The reflections that seemed to be elongated in other diffraction patterns correspond to neighboring reflections of similar intensity. An example is marked by a white ellipse. (c) Diffraction pattern of the approximant in a twofold zone axis. (d) Diffraction pattern of a transition state recorded at a thick sample area showing a pseudofivefold intensity distribution, as outlined by arrows. It was homogenized at $1325 \mathrm{~K}$.

orientation. Diffraction patterns that already show some similarities to a periodic approximant are presented in Figs. 2(c) and 2(d). The diffraction patterns of Figs. 2(b) and 2(c) are similar with respect to additional reflections and elongated reflections but Fig. 2(c) does not resemble a 1D QC. It rather looks as if the reflections are approaching horizontal lines which results in a zigzag pattern of reflections. In Fig. 2(d) the reflections lie on these horizontal lines (the vertical distance corresponds to $3.2 \mathrm{~nm}$ ) with only small deviations of some reflections in the vertical direction. The strongest reflections are strongly elongated in the horizontal direction.

Using large selected-area apertures we observed that weaker reflections merge into streaks along horizontal lines like in Fig. 3(a). Diffraction patterns corresponding to states as represented in diffraction pattern of Fig. 3(a) were observed mainly in furnace-cooled material after homogenization at $1300 \mathrm{~K}$. Approximants free from streaks were only observed in material that was annealed at $1120 \mathrm{~K}$ after homogenization [Fig. 3(b)]. Here it is clearly visible that the strong reflections are split along the horizontal direction. A twofold diffraction pattern of the approximant exhibiting diffuse interlayers is shown in Fig. 3(c). The measured lattice constants of the orthorhombic approximant are $a_{a p}=3.22$, $b_{a p}=5.08$, and $c_{a p}=0.825 \mathrm{~nm}$.

\section{B. Analysis of the HRTEM images}

Figure 4 demonstrates typical features of the transition states observed in this study recorded by HRTEM. It shows the wheel-like structural building units with a diameter of 2 $\mathrm{nm}$ in Scherzer defocus. These building units are usually used as vertices for the construction of tilings. ${ }^{36}$ The tilings are typical for the different structures in the system Al-NiCo; they can be used to analyze the phason strain of the region recorded by HRTEM. In Fig. 4, the pentagonal symmetry is quite pronounced in the center but also at the "spokes" of the wheel-like building units. Regions of equally oriented building units are recognizable in Fig. 4(a). The corresponding tiling is presented in Fig. 4(b).

A magnified detail of Fig. 4(a) is shown in Fig. 4(e). Two tenfold shells, with a diameter of 1.2 and $2 \mathrm{~nm}$, respectively, are the simplified representation of the building units on the right side of Fig. 4(e). The dots on the inner shells represent the fivefold contrast features of the building units. In the HRTEM image a new vertex seems to emerge, indicated by the crossing of the dotted lines on Fig. 4(f). With an edge length $a=2 \mathrm{~nm}$, the distances of neighboring vertices are either $1.2 \mathrm{~nm}$ or $2.0 \mathrm{~nm}$. As a result of the new vertex there are also distances of neighboring vertices with $d$ $=a \sqrt{1+[2 \cos (\pi / 10)]^{-2}}=2.25 \mathrm{~nm}$. These vertex distances result from a different kind of overlap of the building units. For the usual vertex distances the inner or the outer shells touch each other. The new distances are formed when two building units overlap in a way that they have the fivefold contrast features appearing in the HRTEM images in common. Vertices in a distance of $2.25 \mathrm{~nm}$ are connected by dotted lines in all tilings presented in this study.

The appearance of the new vertex distances seems to be coupled with the appearance of kidney shaped motifs in the tilings. The new vertices cannot be indexed according to the tiling with edge length $2 \mathrm{~nm}$. However, it gets clear from the schematic drawing of Fig. 4(f) that the sum of two vectors with $d=2.25 \mathrm{~nm}$ can again be indexed according to the 2 $\mathrm{nm}$ tiling. Building units in a distance of $2.25 \mathrm{~nm}$ are differently oriented. By agglomeration of new distances to lamellar defects as shown in the upper part of the tiling of Fig. 4(b), the tiling cannot be indexed continuously with basic vectors of edge length $2 \mathrm{~nm}$. However, since the sum of two vectors with $d=2.25 \mathrm{~nm}$ is indexable, all vertices separated by an even number of defects can be indexed. Directly at the defects a local periodicity of $3.2 \mathrm{~nm}$ is recognizable.

The projection of all indexable vertices into perpendicular space [Fig. 4(c)] shows an anisotropic distribution; the deviation from a decagonal-pentagonal quasicrystal is already advanced. The jump of coordinates in perpendicular space indicated by an arrow corresponds to positions in physical space where the orientation of the fivefold building units is reversed, a position with occupation inversion. This jump is 


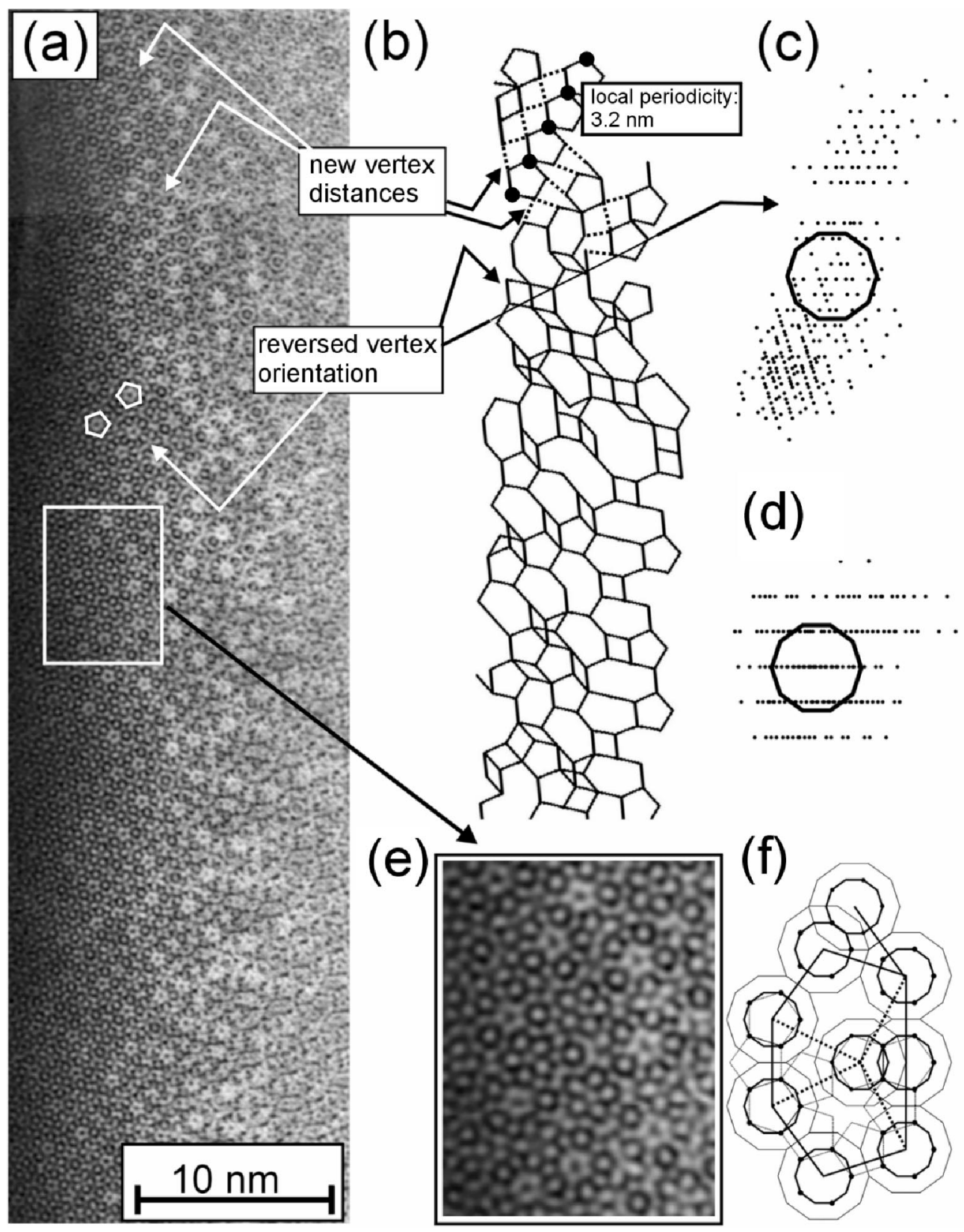

FIG. 4. (a) HRTEM image of material quenched after homogenization at $1310 \mathrm{~K}$. The wheel-like motifs, $2 \mathrm{~nm}$ in diameter, are used as vertices in the corresponding tiling (b). Vertex distances of $2.25 \mathrm{~nm}$ as observed in the upper part of (a) are shown as dotted lines in (b). (c) Projection of the vertices in perpendicular space. (d) Projection of the vertices according to a 1D QC. A kidney-shaped motif of the HRTEM image is enlarged in (e). The overlapping geometry resulting in the new vertex distances is demonstrated in (f).

not observed when the vertices are projected according to the phason strain of a 1D QC. The succession of two lamellar defects with different vertex distances was not observed to be correlated to jumps of coordinates in perpendicular space.

The following tilings are constructed from HRTEM images recorded at the same sample positions as the diffraction patterns shown in previous figures. The tiling corresponding to the diffraction pattern exhibiting S1-superstructure reflections [Fig. 1(a)] is shown in Fig. 5(a). The distribution of vertices in perpendicular space is almost isotropic; some vertices lie out of the acceptance domain of a pentagon Penrose tiling. For more Ni-rich compositions the vertices of tilings representing the S1 superstructure are usually confined to smaller regions in perpendicular space. ${ }^{9,23,37}$ The tiling corresponding to diffraction pattern of Fig. 1(b) is shown in Fig. 6(a). The projection into perpendicular space [Fig. 6(b)] reveals an anisotropic distribution of vertices reflecting deviations from pentagonal-decagonal symmetry. Besides pentagons and rhombi, kidney shaped motifs also appear in the tiling.

The tiling of Fig. 7 shows a good agreement with a 1D $\mathrm{QC}$ as can be seen from the projections in perpendicular space [Fig. 7(b)] and according to the phason strain of a 1D QC [Fig. 7(c)]. The 1D quasicrystalline order is not disturbed by the four lamellar defects indicated by dotted lines. ${ }^{34}$ As in Fig. 4, the defects are not evenly distributed in the tiling. The 


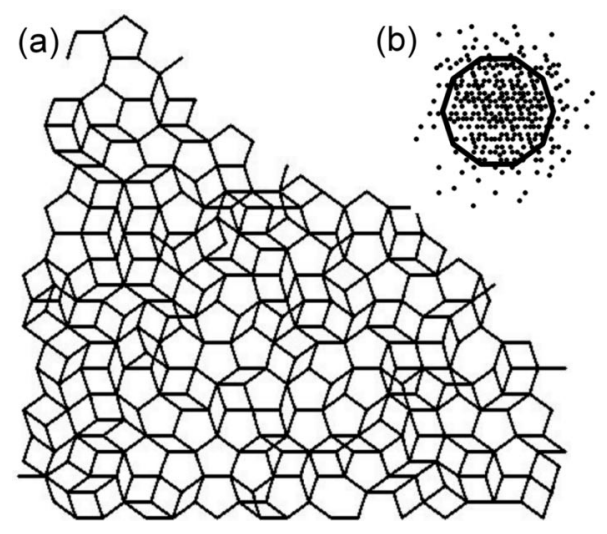

FIG. 5. (a) Tiling with edge length $2 \mathrm{~nm}$ of a region with $\mathrm{S} 1$ superstructure. (b) The projection of the vertices in perpendicular space showing an isotropic distribution.

diffraction pattern of Fig. 2(a) is recorded at the sample region shown on the right side of Fig. 7(a) and the diffraction pattern of Fig. 2(b) comes from the left sample region. The sawtooth pattern of this diffraction pattern is correlated to the appearance of the lamellar defects.

With the accumulated appearance of the lamellar defects the phason strain changes. The order according to a 1D QC disappears and the tiling gets periodic according to $d$ $=3.2 \mathrm{~nm}$. The tiling corresponding to the diffraction pattern of Fig. 2(d) is presented in Fig. 8. In one direction the tiling is periodic with $d=3.2 \mathrm{~nm}$. Perpendicular to this direction periodicity is recognizable in rudiments in the middle part of the tiling.

\section{Non-Fibonacci approximant}

A HRTEM image of a well ordered approximant, corresponding to the diffraction pattern of Fig. 3(b), is shown in Fig. 9. The lamellar defects are ordered periodically as can be seen on the tiling with edge length $2 \mathrm{~nm}$ on the upper part of the figure. The bright dot-like contrasts of the HRTEM image can be used to construct a simplified structure model. A practical choice of tiling has basic vectors in the same orientation as the $2 \mathrm{~nm}$ tiling, deflated by the factor $\tau^{-2}$. This results in edge lengths of $0.76 \mathrm{~nm}$. By choosing this tiling,

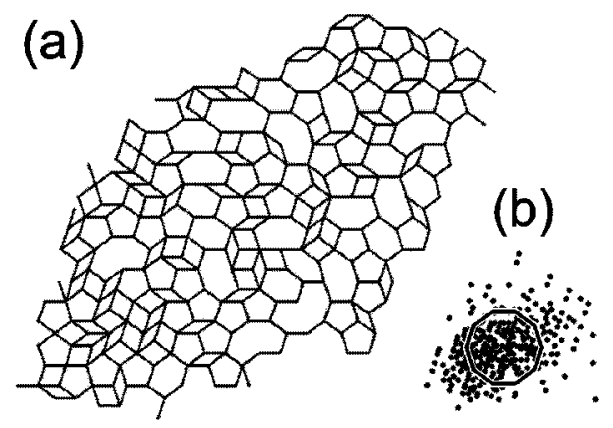

FIG. 6. (a) Tiling, edge length $2 \mathrm{~nm}$, of a HRTEM image coming from the same sample position as the diffraction pattern of Fig. 1. (b) Besides pentagons and rhombi, there appear also kidney shaped motifs in the tiling. The projection of vertices in perpendicular space shows an anisotropic distribution.

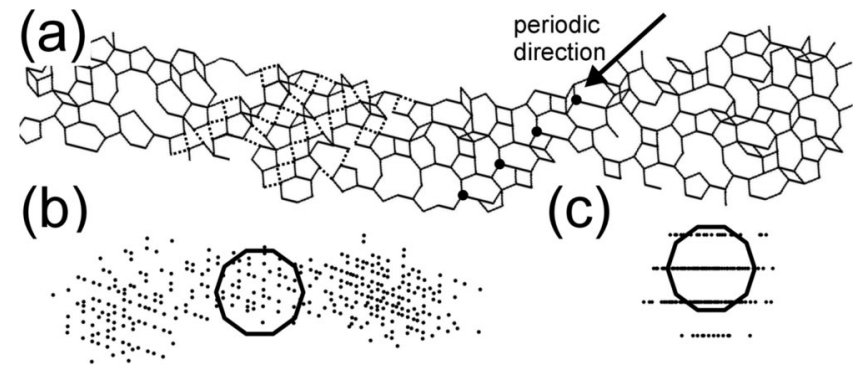

FIG. 7. (a) Tiling with edge length $2 \mathrm{~nm}$ showing lamellar defects indicated by dotted lines. The area on the right side of the tiling corresponds to the diffraction pattern of Fig. 2(a). The area on the left side corresponds to the diffraction pattern of Fig. 2(b). The perpendicular projection of vertices is shown in (b). In (c) the projection according to a $1 \mathrm{D} \mathrm{QC}$ is presented. It shows that the defects do not affect the 1D quasiperiodic order.

the pentagonal contrast features of the building units can be reproduced. However, the tiling cannot be indexed completely in the present case. With each period in the horizontal direction ( $\mathbf{b}_{a p}$ direction) of the approximant there is an additional out-of-phase vector. For a complete indexing of the chosen vertices, basic vectors are needed with an additional deflation by the factor $(2+\tau)^{-0.5}$ (edge length $0.4 \mathrm{~nm}$ ) and a rotation by $90^{\circ}$ with respect to the basic vectors of edge length $0.76 \mathrm{~nm}$. The identity periods in this description are then given by $(0,3,2,-2,-3)$ ( $\mathbf{a}_{a p}$ direction) and $(5,2,-4$, $-4,2) \quad\left(\mathbf{b}_{a p}\right.$ direction). With a further simplification, the elementary cell can be described by a double repetition of a basic motif plus the out-of-phase vector as is shown in the lower part of Fig. 9. The corresponding simulated diffraction pattern is presented in Fig. 10(a). Although a strongly simplified model was used for the simulation, it reflects the splitting of the strong reflections observed in diffraction pattern of Fig. 3(b). Using a model with fourfold repetition of the basic motif plus out-of-phase vector results in the simulated diffraction pattern of Fig. 10(b). In this case the strong reflections seem to be elongated. In between, a large number of weak reflections appear. It is clear that a disturbance of the periodicity would smear out the weak reflections which would result in streaks in the diffraction pattern similar to the experimental diffraction pattern of Fig. 2(d).

The splitting of the strongest reflections into a pair of new ones with roughly equal intensities implies that the indexing

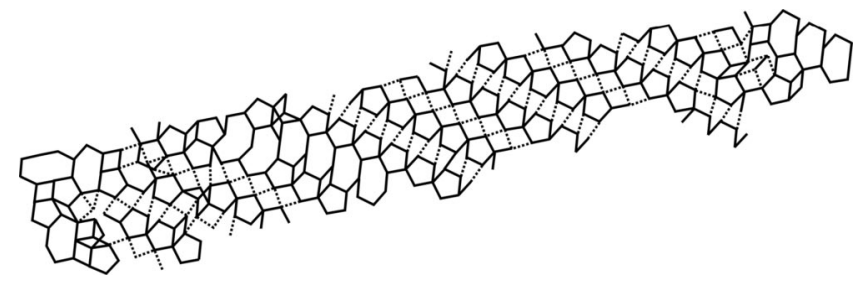

FIG. 8. Tiling, edge length $2 \mathrm{~nm}$, which shows lamellar defects (dotted lines) over the whole region recorded. It is periodic in the direction parallel to the defects with $d=3.2 \mathrm{~nm}$. Perpendicular to this direction there are also first signs of a periodic arrangement in the middle range of the tiling. The tiling is recorded at the same sample region as the diffraction pattern of Fig. 2(d). 


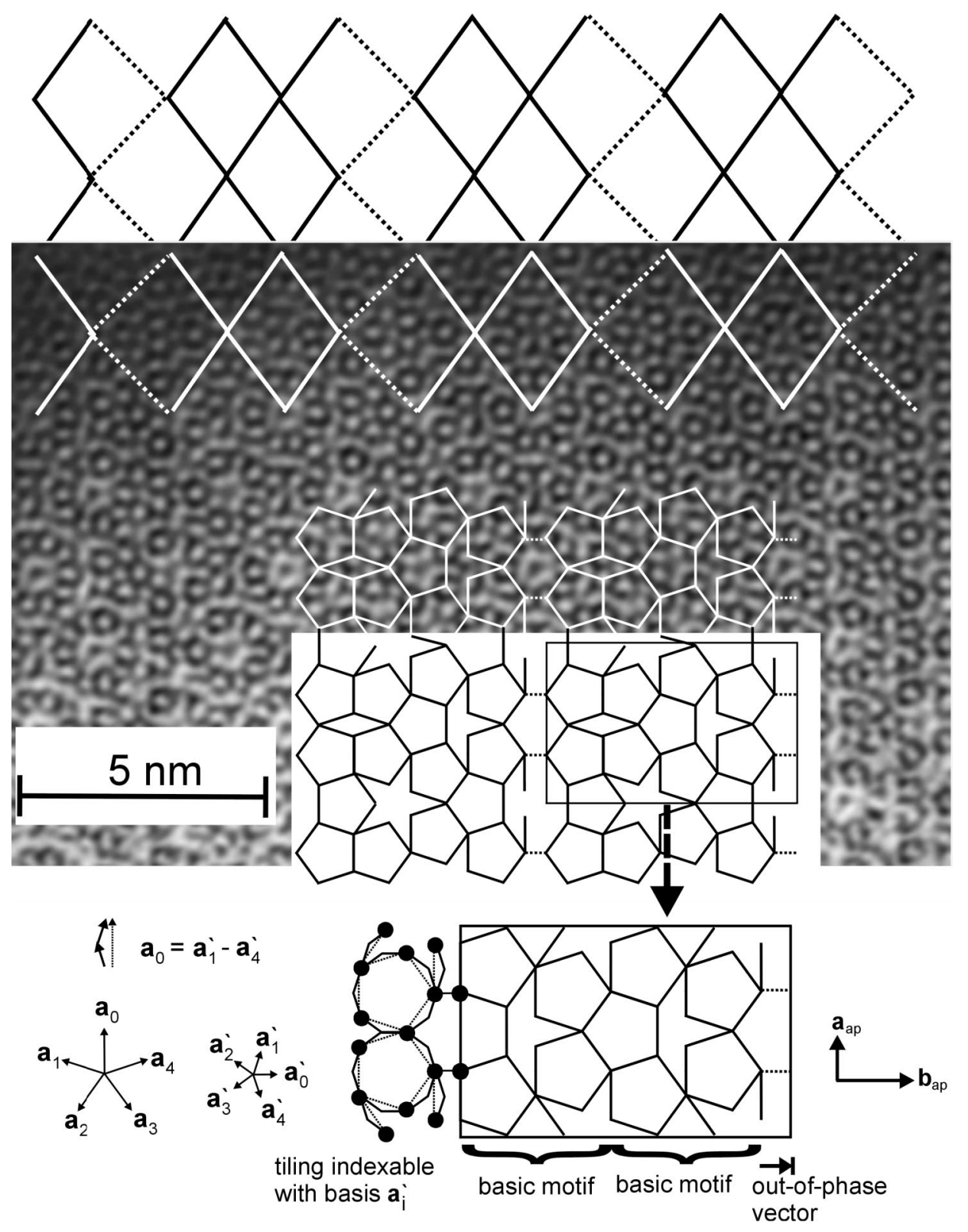

FIG. 9. HRTEM image of the approximant, corresponding to diffraction pattern of Fig. 3(b). The upper tiling, with edge length $2 \mathrm{~nm}$, shows that the lamellar defects are ordered periodically. For the lower tiling drawn in the HRTEM image (edge length 0.76 $\mathrm{nm}$ ), the fivefold contrast features are used for representing the structure. However, it cannot be indexed completely. With a further simplification as shown below, the structure is described by a twofold repetition of a basic structure motif plus an out-of-phase vector. Using a tiling whose orientation is turned around $90^{\circ}$ and an even smaller edge length $(0.4 \mathrm{~nm})$ all vertices can be indexed. In the area of the basic motifs the indexing results always from a sum of two basic vectors.

of the strongest reflections does not necessarily correspond to Fibonacci numbers. Therefore, the present approximant is not of Fibonacci type. This can also be concluded from direct space observations: For Fibonacci approximants, the components of the vectors to the identity period in the direct lattice are determined by two Fibonacci numbers, respectively: $\left(2\left(F_{n}-F_{m}\right), F_{m},-F_{n},-F_{n}, F_{m}\right)$ and $\left(0, F_{r}, F_{s},-F_{s}\right.$, $\left.-F_{r}\right)$, where $m, n, r, s$ are integers. This is not true for $(5,2,-4,-4,2)$. In contrast, the lattice of the basic motif [with the translations $(2,1,-2,-2,1)$ and $(0,3,2,-2,-3)$ ] corresponds to a Fibonacci approximant, the so-called $\mathrm{PD}_{2}{ }^{22}$ This shows that in this case, the formation of the nonFibonacci approximant is related to the formation of out-ofphase domains.

When the indexing of the next neighbors of a vertex changes with respect to the basic vectors with $0.76 \mathrm{~nm}$ edge length by $\mathbf{a}_{i}$, then the indexing with respect to the basic vectors with $0.4 \mathrm{~nm}$ edge length changes by $\mathbf{a}_{i+1}^{\prime}{ }_{\bmod 5}$ $-\mathbf{a}_{i+4_{\bmod 5}^{\prime}}$. Therefore, the sum of all components of vertices is the same within the basic motifs. However, with each period in the $\mathbf{b}_{a p}$ direction the sum changes by one because of the out-of-phase vector. Hence, the acceptance domain makes a jump by each period in the $\mathbf{b}_{a p}$ direction. In contrast, for Fibonacci approximants the equations $\sum_{i=1}^{5} p_{i}$ $=\sum_{i=1}^{5} q_{i}=0$ hold for the periods $\mathbf{p}$ and $\mathbf{q}$.

\section{DISCUSSION}

Based on the experimental observations, the following scenario for the transformation process and the sequence of intermediate states, schematically shown in Fig. 11, can be developed.

The transition starts with increasing linear phason strain resulting in a $1 \mathrm{D}$ QC. The building units show pentagonal symmetry; they are equally oriented within domains. These 


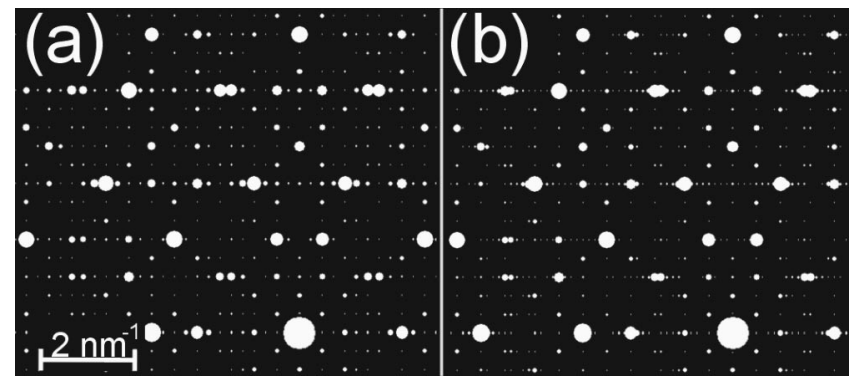

FIG. 10. (a) Simulated diffraction pattern with the simplified model of Fig. 9. It reproduces neighboring reflections with similar intensities. (b) Simulated diffraction pattern with fourfold repetition of the basic structure motif plus out-of-phase vector. The split main reflections look due to their short distance as if they were elongated. Similar to Fig. 2(d) there are further weak reflections recognizable.

domains can be regarded as twin domains that could develop, for example, when the decagonal symmetry gets lost by cooling the material. In the tiling kidney-shaped motifs appear, allowing an unusual kind of overlap of building units. This overlap is assumed to be related to the fivefold character of the building units, as two adjacent building units have the fivefold contrast features of the "spokes" in common. This results in the occurrence of the vertex distance $2.25 \mathrm{~nm}$. With increasing phason strain vertices in this distance agglomerate to lamellar defects and out-of-phase domains are formed which results in split reflections in the diffraction patterns. The domains are preferentially oriented in a way that allows a period of $3.2 \mathrm{~nm}$ at the boundary plane. The increasing number of lamellar defects destroys the order of the 1D QC and enforces a periodicity of $3.2 \mathrm{~nm}$. A periodic arrangement of the out-of-phase domains leads to the formation of a periodic approximant.

The low-temperature ordering state without superstructure reflections and pseudofivefold symmetry known as " $5 f$ ", 11,27 belongs to the first part of the transition. It should be considered as a transition state of a pentagonal or a decagonal quasicrystal which exists at temperatures close to the solidus. ${ }^{29}$ Whether the approximant is stable or only a transition state prior to the formation of normal-crystalline phases cannot be decided from our experiments. The kinetic barriers for the

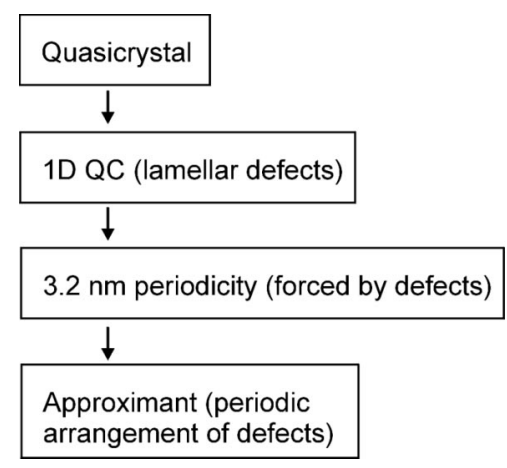

FIG. 11. Model for the sequence of states occurring in the course of the observed transition. formation of the approximant can be expected to be much lower than for the formation of normal-crystalline phases. For example, in $\mathrm{Al}_{72.5} \mathrm{Ni}_{12} \mathrm{Co}_{15.5}$ it was observed ${ }^{9}$ that a metastable NDS (known earlier as SST-II) decomposed after annealing for almost $3000 \mathrm{~h}$ into a quasicrystal and the crystalline $X$ phase. It might be worthwhile to note that at the studied composition, twinned nanodomains with different directions of linear phason strain as is the case for the usual NDS's (Refs. 7-9) were not observed.

The observed transition by the formation of out-of-phase domains seems to be a special case among the transitions between quasicrystals and approximants. Transitions including twinned phason strained NDS's as transition states transform differently also at later stages of the transition because the transition is discontinuous regarding phason strain. Untwinned phason strained states usually resulting from material quenched from the melt also appear to be different. For these states, out-of-phase domains are not reported, let alone periodic ordering of out-of-phase domains. This might be related to the fact that these transitions were not observed to result in non-Fibonacci approximants. However, lamellar defects that can be considered to form out-of-phase domains are also possible for transition states of Fibonacci approximants. In this case, the lamellae are rather formed by Fibonacci-type rhombi. ${ }^{38}$ Usually, these defects are even difficult to remove by annealing. A similar role of out-of-phase domains in the process of a transition between quasicrystal and approximant has not been reported yet. However, according to our unpublished results, similar transition states appear also in the system $\mathrm{Al}-\mathrm{Cu}-\mathrm{Co}$, indicating a transferability of the results presented in this study.

The approximant observed in this study and the nonFibonacci approximant described by Sui et al. ${ }^{12}$ differ regarding important features. The latter is built of thick and thin rhombi with an edge length of $0.66 \mathrm{~nm}$. There is no clear indication of an additional out-of-phase vector. Consequently, the strong reflections are not split in this case.

This transition is on the one hand a continuous transition because the phason strain direction is the same over large distances. Despite this fact, the structure can be regarded as some kind of NDS's because twin domains (with different orientations of fivefold building units) and out-of-phase domains in the range of nanometers are formed. In the case of a transition by phason strained nanodomains it is assumed that a misfit between a periodic and quasiperiodic structures increases with the size of the transformed region. ${ }^{39}$ Therefore, the formation of small periodic domains could be favorable. The question whether the formation of the out-ofphase domains locally reduces jumps or shifts of atoms in the process of the transition must be evaluated in a more detailed study.

\section{SUMMARY}

The transition between quasicrystal and approximant on differently annealed samples with a nominal composition of $\mathrm{Al}_{72.7} \mathrm{Ni}_{8.3} \mathrm{Co}_{19}$ is observed by electron diffraction and HRTEM. The transition starts with increasing phason strain resulting in an untwinned one-dimensional quasicrystal. In the 
course of this process a previously unknown overlap of building units is observed which is related to the fivefold character of the building units. This results in vertex positions not indexable by tiles with an edge length of $2 \mathrm{~nm}$. Out-of-phase domains are formed by agglomeration of such vertices to lamellar defects. In the following, the order according to a one-dimensional quasicrystal is lost because these lamellae enforce a periodicity of $3.2 \mathrm{~nm}$ at their bound-

*Present address: Robert Bosch GmbH, GS/EVT4, Postfach 300240, D-70442 Stuttgart, Germany.

${ }^{1}$ A. P. Tsai, in Physical Properties of Quasicrystals, edited by Z. M. Stadnik (Springer-Verlag, Berlin, 1999), pp. 5-50.

${ }^{2}$ A. I. Goldman and K. F. Kelton, Rev. Mod. Phys. 65, 213 (1993).

${ }^{3}$ M. Duneau and A. Katz, Phys. Rev. Lett. 54, 2688 (1985).

${ }^{4}$ V. Elser and C. L. Henley, Phys. Rev. Lett. 55, 2883 (1985).

${ }^{5}$ H. Zhang and K. H. Kuo, Phys. Rev. B 41, 3482 (1990).

${ }^{6}$ J. M. Dubois, C. Dong, C. Janot, M. de Boissieu, and M. Audier, Phase Transit. 32, 3 (1991).

${ }^{7}$ M. Fettweis, P. Launois, F. Dénoyer, R. Reich, and M. Lambert, Phys. Rev. B 49, 15573 (1994).

${ }^{8}$ M. Kalning, S. Kek, H. Krane, V. Dorna, W. Press, and W. Steurer, Phys. Rev. B 55, 187 (1997).

${ }^{9}$ M. Döblinger, R. Wittmann, and B. Grushko, Phys. Rev. B 64, 134208 (2001)

${ }^{10}$ K. Edagawa, K. Suzuki, M. Ichihara, S. Takeuchi, and T. Shibuya, Philos. Mag. B 64, 629 (1991).

${ }^{11}$ W. Steurer and T. Haibach, in Physical Properties of Quasicrystals, edited by Z. M. Stadnik (Springer-Verlag, Berlin, 1999), pp. $51-89$.

${ }^{12}$ H. X. Sui, X. Z. Liao, and K. H. Kuo, Philos. Mag. Lett. 71, 139 (1995).

${ }^{13}$ H. X. Sui, X. Z. Liao, K. H. Kuo, X. Zou, and S. Hovmöller, Acta Crystallogr., Sect. B: Struct. Sci. 53, 587 (1997).

${ }^{14}$ F. Frey, in Local Structure from Diffraction, edited by S. J. L. Billinge and M. F. Thorpe (Plenum Press, New York, 1998), pp. 295-321.

${ }^{15}$ F. Frey, Mater. Sci. Eng., A 294-296, 178 (2000).

${ }^{16}$ H. Sato and R. Toth, Phys. Rev. 124, 1833 (1961).

${ }^{17}$ T. Janssen and A. Janner, Adv. Phys. 36, 519 (1987).

${ }^{18}$ A. T. Paxton and H. M. Polatoglou, Phys. Rev. Lett. 78, 270 (1997)

${ }^{19}$ K. Edagawa, M. Ichihara, K. Suzuki, and S. Takeuchi, Philos. Mag. Lett. 66, 19 (1992).

${ }^{20}$ B. Grushko and K. Urban, Philos. Mag. B 70, 1063 (1994).

${ }^{21}$ S. Ritsch, C. Beeli, H.-U. Nissen, T. Gödecke, M. Scheffer, and R. Lück, Philos. Mag. Lett. 78, 67 (1998).

${ }^{22}$ B. Grushko, D. Holland-Moritz, R. Wittmann, and G. Wilde, J. Alloys Compd. 280, 215 (1998).

${ }^{23}$ S. Ritsch, Ph.D. thesis, ETH Zürich, 1996. ary plane. By annealing, a periodic arrangement of the lamellae can be achieved and a non-Fibonacci approximant is formed.

\section{ACKNOWLEDGMENTS}

We thank V. Zibat for microprobe analysis. This investigation was financially supported by the Deutsche Forschungsgemeinschaft under Contract No. Wi1645/1-2.

${ }^{24}$ M. Kalning, S. Kek, B. Burandt, W. Press, and W. Steurer, J. Phys.: Condens. Matter 6, 6177 (1994).

${ }^{25}$ E. Weidner, F. Frey, and K. Hradil, Philos. Mag. A 81, 2375 (2001).

${ }^{26}$ T. Gödecke, M. Scheffer, R. Lück, S. Ritsch, and C. Beeli, Z. Metallkd. 89, 687 (1998).

${ }^{27}$ S. Ritsch, C. Beeli, and H.-U. Nissen, Philos. Mag. Lett. 74, 103 (1996).

${ }^{28}$ S. Ritsch, C. Beeli, R. Lück, and K. Hiraga, Philos. Mag. Lett. 79, 225 (1999).

${ }^{29}$ A. Baumgarte, J. Schreuer, M. A. Estermann, and W. Steurer, Philos. Mag. A 75, 1665 (1997).

${ }^{30}$ A. Yamamoto, Acta Crystallogr., Sect. A: Found. Crystallogr. 52, 509 (1996)

${ }^{31}$ S. Ritsch, O. Radulescu, C. Beeli, D. Warrington, R. Lück, and K. Hiraga, Philos. Mag. Lett. 80, 107 (2000).

${ }^{32}$ T. C. Lubensky, J. E. S. Socolar, P. J. Steinhardt, P. A. Bancel, and P. A. Heiney, Phys. Rev. Lett. 57, 1440 (1986).

${ }^{33}$ Due to spherical aberration, the use of very small apertures for diffraction is critical for beams far away from the optic axis. As a consequence, areas of the sample that are not selected by the aperture contribute to the diffraction image. That applies especially for large reciprocal vectors whose diffraction angle $2 \theta_{0}$ is large. For the displacement distance $r$ holds: $r=C_{s}\left(2 \theta_{0}\right)^{3}$. However, the largest reciprocal space vectors shown in our diffraction patterns correspond to distances of about $0.2 \mathrm{~nm}$. This corresponds to a displacement distance is smaller than $2.5 \mathrm{~nm}$ for an acceleration voltage of $200 \mathrm{kV}$.

${ }^{34}$ The vertices after the first and the third defect are not indexed.

${ }^{35}$ K. Hiraga, T. Ohsuna, and S. Nishimura, Philos. Mag. Lett. 81, 123 (2001).

${ }^{36} \mathrm{~K}$. Hiraga, in Quasicrystals. The State of the Art., edited by D. P. DiVincenzo and P. J. Steinhardt (World Scientific, Singapore, 1991), pp. 95-110.

${ }^{37}$ K. Edagawa, H. Tamaru, S. Yamaguchi, K. Suzuki, and S. Takeuchi, Phys. Rev. B 50, 12413 (1994).

${ }^{38}$ M. Döblinger, R. Wittmann, D. Gerthsen, and B. Grushko, Mater. Sci. Eng., A 294-296, 131 (2000).

${ }^{39}$ W. Steurer, in Quasicrystals, edited by J. M. Dubois, P. A. Thiel, A. P. Tsai, and K. Urban, Mater. Res. Soc. Symp. Proc. No. 553 (Materials Research Society, Pittsburgh, 1999), p. 159. 